\title{
Articular cartilage in corn oil-induced lipoarthrosis
}

\author{
P. N. MEHTA* AND F. N. GHADIALLY \\ From the Department of Pathology, University Hospital Saskatoon, Saskatchewan, Canada
}

Injury to a joint can lead not only to the liberation of blood (haemarthrosis) from ruptured vessels but also of lipid (lipohaemarthrosis) from juxta-articular fat pads when the synovial membrane is torn or from the bone marrow when an intra-articular fracture has occurred. The manner in which blood shed into the joint is removed and the pathological changes that occur in synovial membrane and cartilage in acute and chronic haemarthrosis in man (Key, 1932; Roy and Ghadially, 1967; Ghadially and Roy, 1969a) and experimental animals (Key, 1929; Ghadially and Roy, 1967, 1969a; Hoaglund, 1967; Roy and Ghadially, 1966, 1969) have been extensively studied. There is good reason to believe that in lipohaemarthrosis too, the haemic component is removed in a similar manner (Ghadially and Roy, 1969b), but the fate of the lipidic component and its effect on articular tissues is poorly understood.

We have now carried out some investigations on this problem and have shown that in human lipohaemarthrosis large accumulations of electron lucent lipid occur in the synovial intimal cells (Ghadially and Roy, 1969b) and that an identical appearance can be produced in the synovial membrane of the rabbit knee joint by injections of autologus fat derived from the omentum (Ghadially, Janzen, and Mehta, 1970). Perhaps even more interesting is the observation that autologus fat injected into the joint seems readily to enter articular cartilage and chondrocytes, producing changes which suggest that the health of articular cartilage is adversely affected by such a procedure (Ghadially, Mehta, and Kirkaldy-Willis, 1970).

In human lipohaemarthrosis the lipid shed into the joint is derived from the juxta-articular fat pads or bone marrow. In our experimental animals it was derived from the omentum. In each instance we were looking at storage or depot fat which, as is well known, is composed largely or almost entirely of triglycerides of unsaturated fatty acids. Such fat is non-osmiophilic or feebly osmiophilic and in electron micrographs its presence is indicated by electron lucent areas of very low density (Stockenius and Mahr, 1965; Fawcett, 1966). Tracking the passage of such material from joint space to chondrocyte is difficult and, since a fair number of electron lucent lipid droplets occur normally in chondrocytes, there is always the danger of confusing these with those derived from the injected lipid.

Therefore, it seemed worthwhile to repeat our experiments using an osmiophilic lipid, namely corn oil (Mazola), wherin approximately 86 per cent. of the total fatty acid content is unsaturated (Table).

\section{Table Corn oil constituents (per cent.)*}

\begin{tabular}{|c|c|}
\hline $\begin{array}{l}\text { Glycerides } \\
\text { Unsaponifiable matter } \\
\text { Total } \\
\text { Sitosterols }\end{array}$ & $\begin{array}{l}98 \cdot 6 \\
1.4(1 \cdot 26-1 \cdot 63) \\
1.0(0.92-1.08)\end{array}$ \\
\hline $\begin{array}{c}\text { Fatty acid distribution } \\
\text { total acids) } \\
\text { Saturated } \\
\text { Palmitic } \\
\text { Stearic }\end{array}$ & $\left.\begin{array}{r}10 \\
3\end{array}\right\} 13$ \\
\hline $\begin{array}{l}\text { Unsaturated } \\
\text { Linoleic } \\
\text { Oleic }\end{array}$ & $\left.\begin{array}{l}56 \\
30\end{array}\right\} 86$ \\
\hline
\end{tabular}

* Data obtained from booklet entitled 'Corn Oil', (1960), 2nd ed., p. 13 Published by Corn Industries Research Foundation, Inc., 1001 Connecticut Ave., N.W., Washington 6, D.C.

We felt that such a lipid would be electron dense and could hence be tracked more confidently with the electron microscope. Furthermore, if electron dense lipid droplets could be demonstrated in the chondrocytes after the injection of corn oil into the joint. unequivocal proof of entry of lipid from joint space to cartilage would be obtained, for markedly electron dense lipid droplets are rarely, if ever, seen in the articular cartilage of this animal.

\section{Materials and methods}

Twelve 3- to 4-month-old rabbits weighing between 2 and $3 \mathrm{~kg}$. were used in this experiment. Under Nembutal anaesthesia approximately $1.5 \mathrm{ml}$. of corn oil was injected with strict aseptic precautions into the right knee joint. 
Two or three animals were killed thereafter by intracardiac injection of sodium pentothal at each of the following intervals: 24 hrs, 2 days, 4 days, 5 days, and 1 week. Articular cartilage from both the right and the left knee joint was collected immediately after and processed.

\section{PROCESSING OF TISSUES}

A thin shaving of cartilage obtained from the weightbearing area of the lateral femoral condyle was placed immediately in cold 2 per cent. osmium buffered with cacodylate. After $1 \frac{1}{2}$ hrs' fixation the piece of cartilage was trimmed into strips about 3 to $4 \mathrm{~mm}$. long and $1 \mathrm{~mm}$. wide. Tissues were then dehydrated in increasing concentrations of ethanol, cleared in propylene oxide, embedded in Epon, and cut with diamond knives in Porter-Blum or Reichert microtomes. Ultrathin sections 500 to $600 \AA$ thick were mounted on copper grids, stained with uranyl acetate and lead hydroxide, and examined with the electron microscope (Zeiss EM-9S or RCA EMU-3G).

For light microscopy, tissues were fixed in 10 per cent. formalin, embedded in paraffin, and stained with haematoxylin and eosin. Frozen sections were also made and stained with oil red O. Semi-thin sections $(0.5$ to $1 \mu)$ were cut from all the Epon blocks, stained with Richardson's stain (Richardson Jarett, and Finke, 1960), and examined with the light microscope.

\section{Results}

\section{Macroscopic examination}

Injected lipid could be readily identified within the joint cavity as droplets or a milky emulsion up to the second day. A trace of such material was detectable on the fourth day but neither frank lipid nor an emulsion was detectable at later time intervals.

\section{Light microscopy}

The examination of the sections stained with haematoxylin and eosin or oil red 0 did not prove very helpful. However, it is perhaps worth noting that droplets of lipid could be demonstrated within the chondrocytes as fine red refractile material. Any changes seen in these could be more accurately and confidently demonstrated in the semi-thin sections from the Epon blocks. There the lipid presented as innumerable fine, round, clear or greyish-yellow, punched-out areas within the cytoplasm of chondrocytes. In some sections the lipid droplets appeared to stain a light blue colour.

The distribution of intracellular lipid in the chondrocytes of control joints was similar to that previously reported (Collins, Ghadially, and Meachim, 1965); that is to say only an occasional Zone I and II cell contained lipid but Zone III chondrocytes contain lipid more often (Fig. 1).

In specimens of cartilage obtained 1 day after the injection of corn oil, there was a clear-cut increase in the number of lipid droplets in Zone I and II chondrocytes. Maximum increase was seen in 2-day specimens (Fig. 2), where virtually every chondrocyte contained one or many lipid droplets. In 4-day specimens the lipid was still plentiful but not as marked as at 2 days, while in 1-week specimens the lipid content was much reduced being little or no more than that in normal articular cartilage.

Another change noted was the occurrence of numerous large clear lacunae which were either empty or contained remnants of disintegrating chondrocytes. Such in situ necrosis of chondrocytes is a normal phenomenon and was also found if control specimens but much more in situ necrosis was evident in the injected joints. Furthermore, in some articular cartilage, the surface was uneven and in some animals the continuity of the articular surface was interrupted (Fig. 2). This could be interpreted as evidence of early fibrillation.

\section{Electron microscopy}

Articular cartilage from control knees was essentially similar to normal rabbit cartilage described by us and

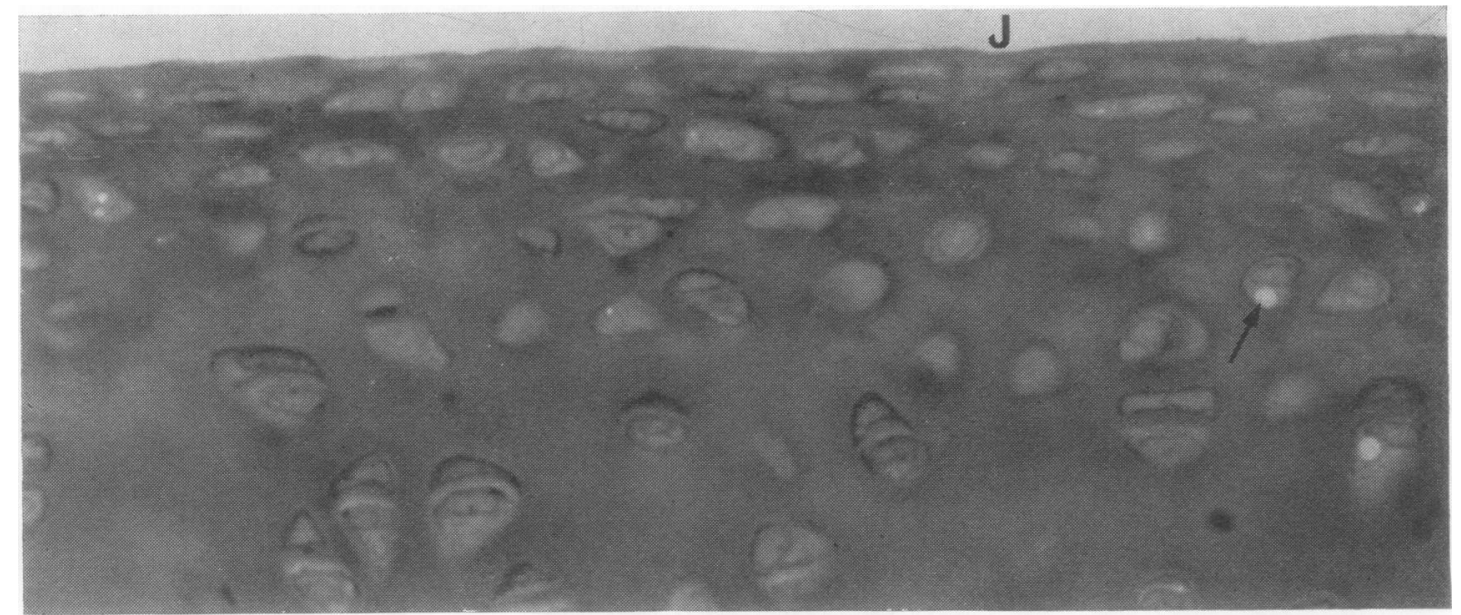

FIG. 1 Normal articular cartilage (for comparison with Fig. 2) showing smooth surface adjacent to joint space (J) $A$ few chondrocyte contain lipid droplets (arrow), which appear as 'holes' within the cells. Richardson's stain. $\times 700$ 


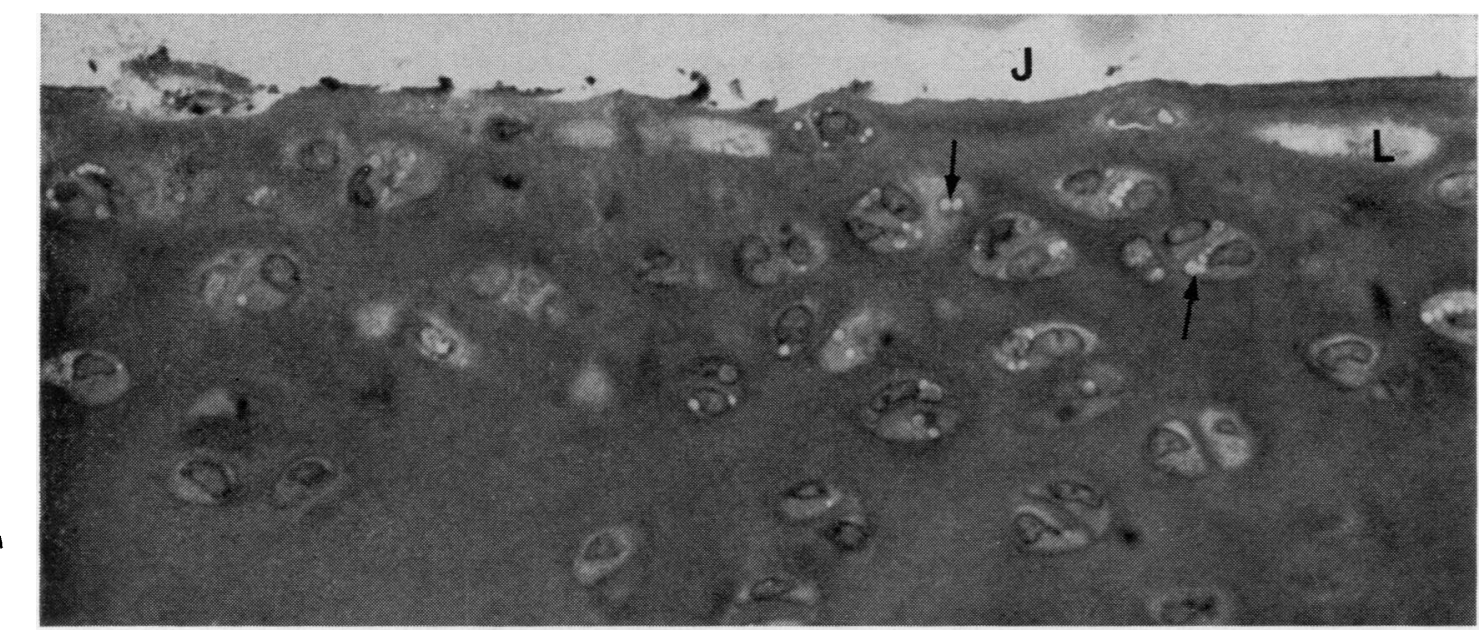

FIG. 2 Articular cartilage 2 days after corn oil injection. Most of the chondrocytes of the superficial and transitional zones contain one or more lipid droplets (arrows). Also seen are some large lacunae (L) and an irregular and discontinuous articular surface adjacent to the joint space $(J)$. Richardson's stain. $\times 700$

others (Davies, Barnett, Cochrane, and Palfrey, 1962; Barnett, Cochrane, and Palfrey, 1963; Palfrey and Davis, 1966; Ghadially and Roy, 1969a). The sequence of events that occurred in the experimental joints has already been described; the ultrastructural changes can therefore be considered collectively.

By electron microscopy we confirmed the light microscopic finding of numerous lipid droplets in chondrocytes. Here the lipid occurred as large or small rounded masses lying within the cytoplasmic matrix. The electron density of the lipid varied from specimen to specimen (Figs 3 and 4). Even so it was invariably much more dense than the electron lucent lipid seen in chondrocytes after the injection of autologous fat into the joint (Fig. 5). A close correlation between the degree of electron density and the time interval at which the specimen was collected could not be established, but it seemed that maximum density lipid occurred at early time intervals (1 to 4 days) and there was as a rule a marked fading of electron density in later specimens ( 5 days and 1 week). At times Golgi vacuoles and vesicles also contained electron dense material similar in appearance to the cytoplasmic lipid droplets (Fig. 6). This phenomenon was witnessed mainly in the 2-day specimens and was not seen at later time intervals ( 5 days and 1 week) or in any of the cartilage samples from the control joints.

The cell membranes, organelles, and inclusions of the chondrocytes also showed some changes that are worth noting. Frequently the cell processes did not extend out into the matrix but were folded parallel to

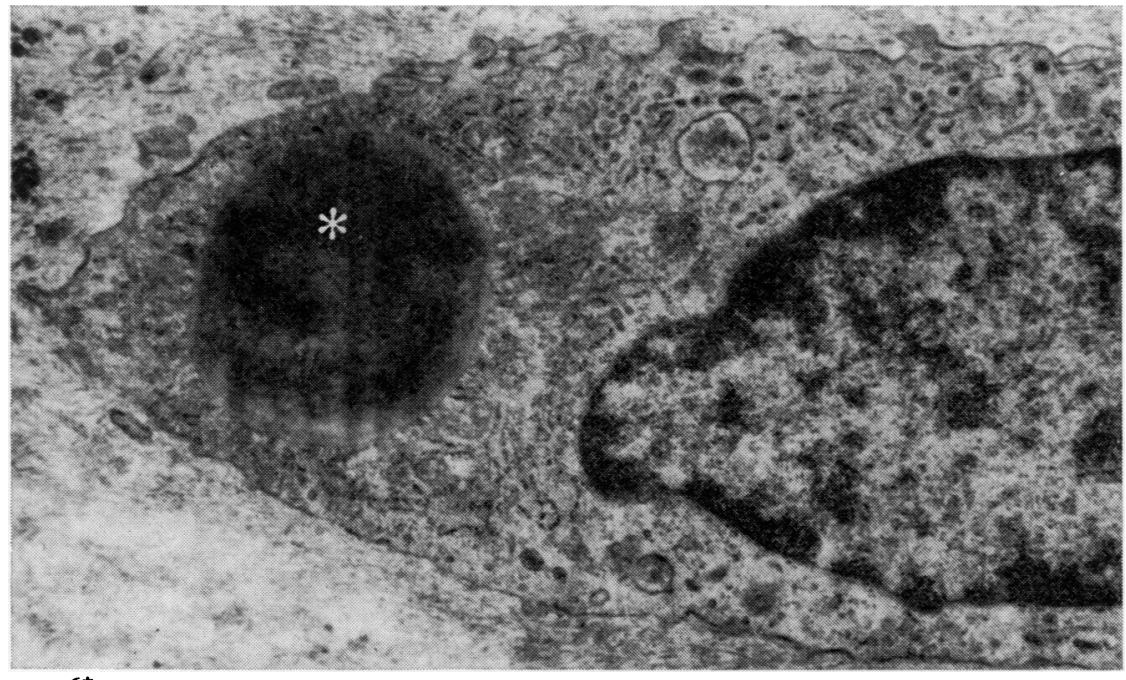

FIG. 3 Chondrocyte from corn oil-injected 4 joint, showing a moderately electron dense lipid droplet $\left(^{*}\right) . \times 13,000$ 


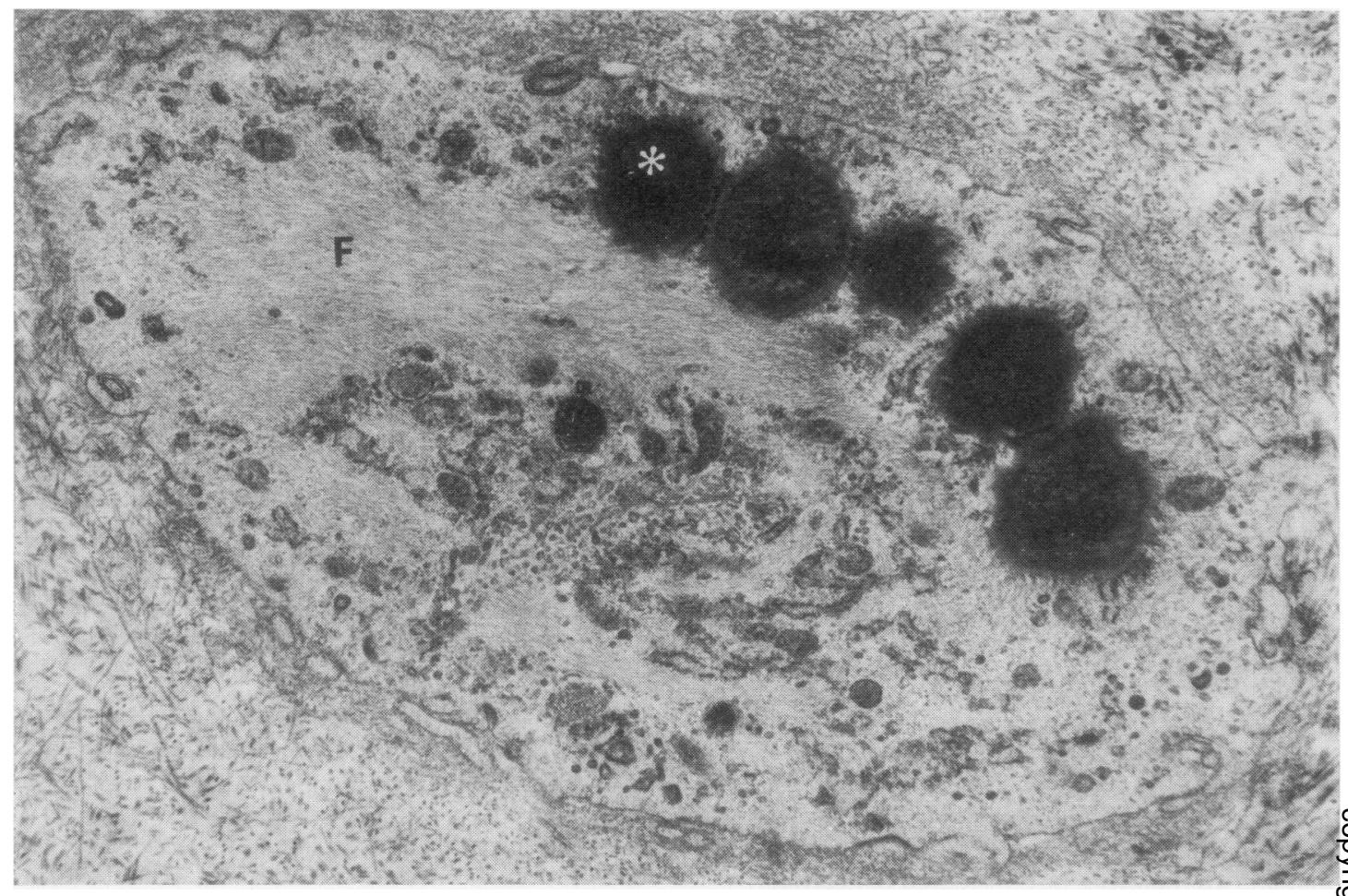

FIG. 4 Chondrocyte from corn oil-injected joint, showing a group of highly electron dense lipid droplets $\left({ }^{*}\right)$. Note als $\bar{Q}$ paucity of organelles and abundance of fine intracytoplasmic filaments $(F) . \times 13,000$

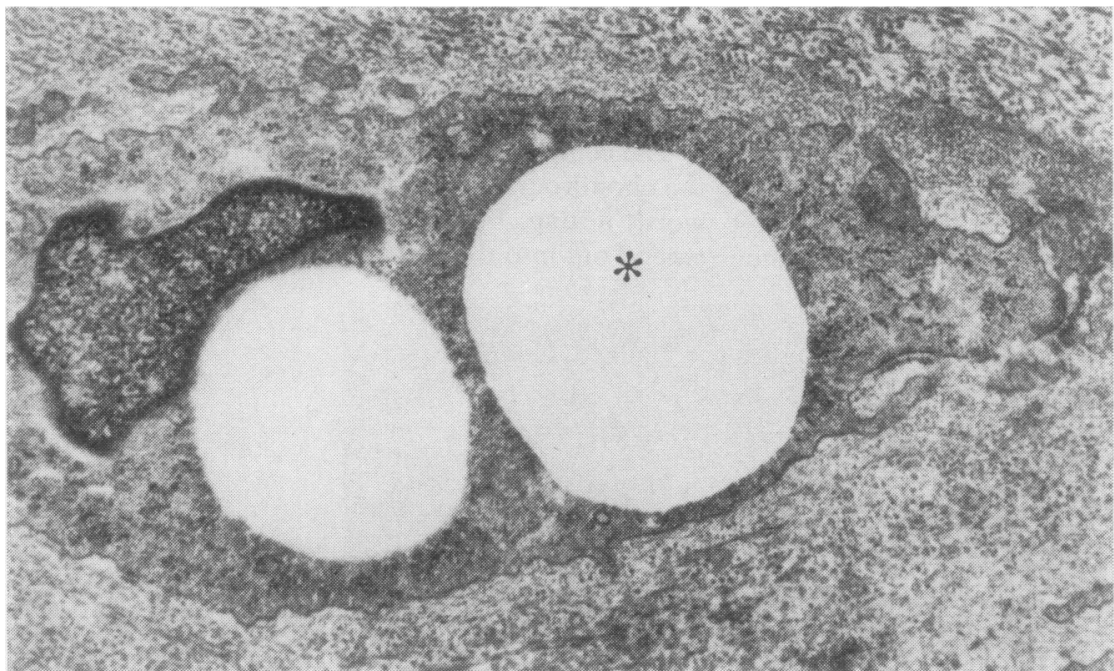

FIG. 5 Chondrocyte from autologous fat-injected joint ( for comparison with Figs 3 and 4), showing electron lucent droplets $\left({ }^{*}\right) . \times 16,000$

the cell surface. Micropinocytotic vesicles were scanty and in no instance could we find any evidence that lipid was being transported into the cell by these structures. There was a relative paucity of glycogen but fine filamentous fibres were much more abundant in chondrocytes of experimental than control joints (Fig. 4). In the intact chondrocytes the other organelles did not show any noteworthy changes. Chondro- cytes showing in situ necrosis were frequently encountered, and it was evident that disintegration of cells at times liberated the corn oil-derived, electron dense lipid droplets into the cartilage matrix (Fig. 7).

In our attempt to track the osmiophilic lipid from joint space to chondrocyte we carefully searched the matrix for electron dense droplets, but we found no gradient of fine electron dense lipid droplets extending 


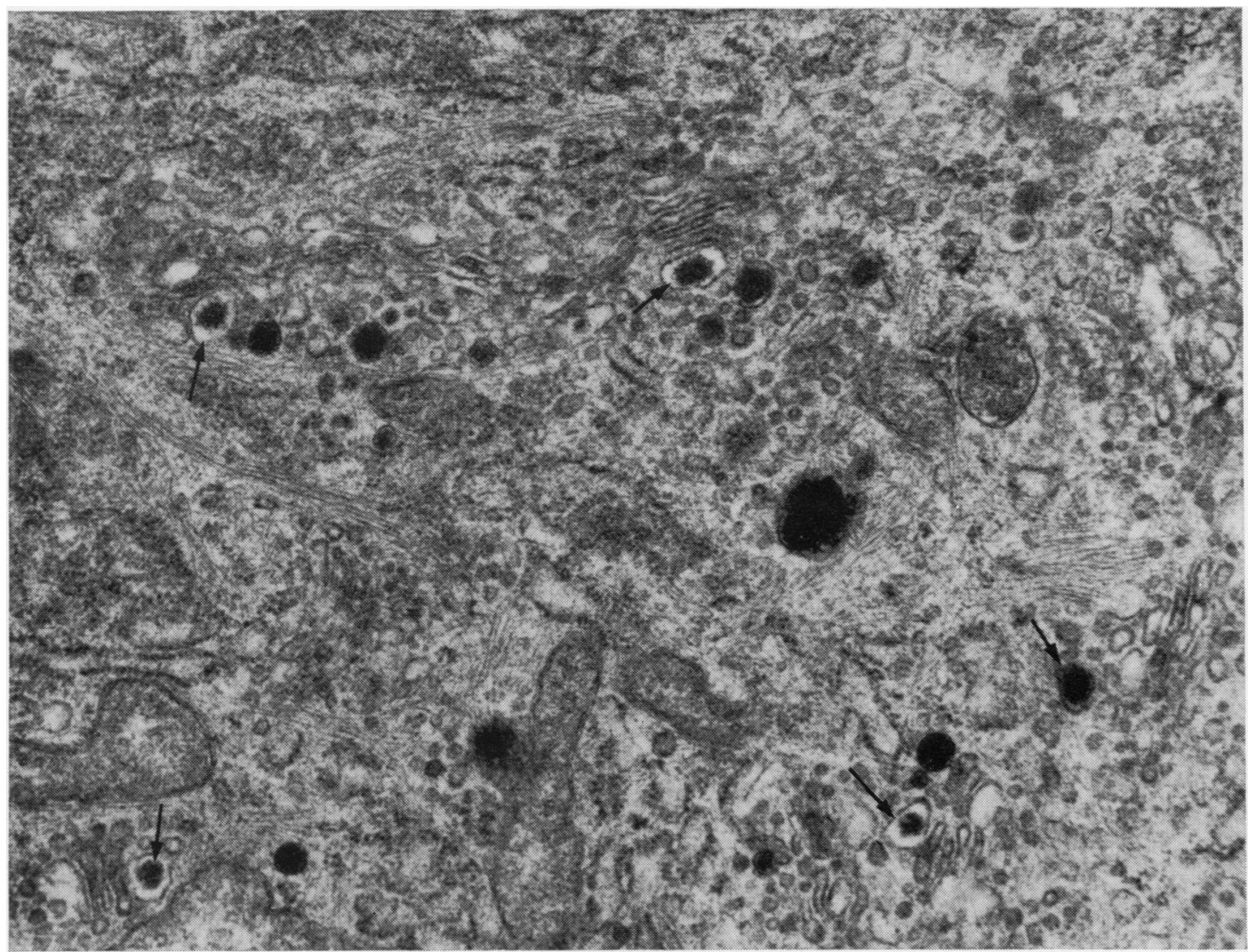

FIG. 6 Chondrocyte 2 days after corn oil injection. Electron dense lipidic material (arrows) is seen in vacuoles and vesicles of the Golgi complex. $\times 32,000$

from the articular surface to the deeper zones of cartilage as one might have expected. However, electron dense lipid droplets were at times seen in the matrix (Fig. 8) admixed with membraneous and granular debris of the type derived by in situ disintegration of chondrocytes (Ghadially, Meachim, and Collins, 1965).

\section{Discussion}

The results of our present experiment confirm and amplify the findings of our previous study where autologous fat was injected into rabbit joints (Ghadially and others, 1970). Collectively these studies show that, when a saturated fatty acid rich lipid (autologous fat) is placed in the joint, the chondrocytes are filled with innumerable electron lucent lipid droplets, but if an unsaturated fatty acid rich lipid (corn oil) is used the chondrocytes become loaded with droplets of medium and high electron density. Thus there can be little doubt that fat lying in the joint space readily enters articular cartilage and that the lipid seen in the chondrocytes is injected lipid and not normally occurring lipid which has accumulated in the chondrocyte as a result of some metabolic derangement engendered by the experimental procedure.

Two problems are posed by our experiments:

(1) How does the lipid traverse the matrix?

(2) How is it taken up by chondrocytes?

The notion that lipid may travel as fine droplets through the matrix is not supported by our results, for we did not find such osmiophilic droplets scattered in the matrix nor did we observe a gradient of droplets from surface to interior. In previous experiments, where autologous lipid was used, a similar result was obtained, but we felt that this might be due to the technical difficulty of tracking an electron lucent material. It seems now that, since osmiophilic lipid which should be trackable is also not seen crossing the matrix in this manner, one has to concede either that the lipid traverses the matrix as submicroscopic aggregates of triglyceride molecules 


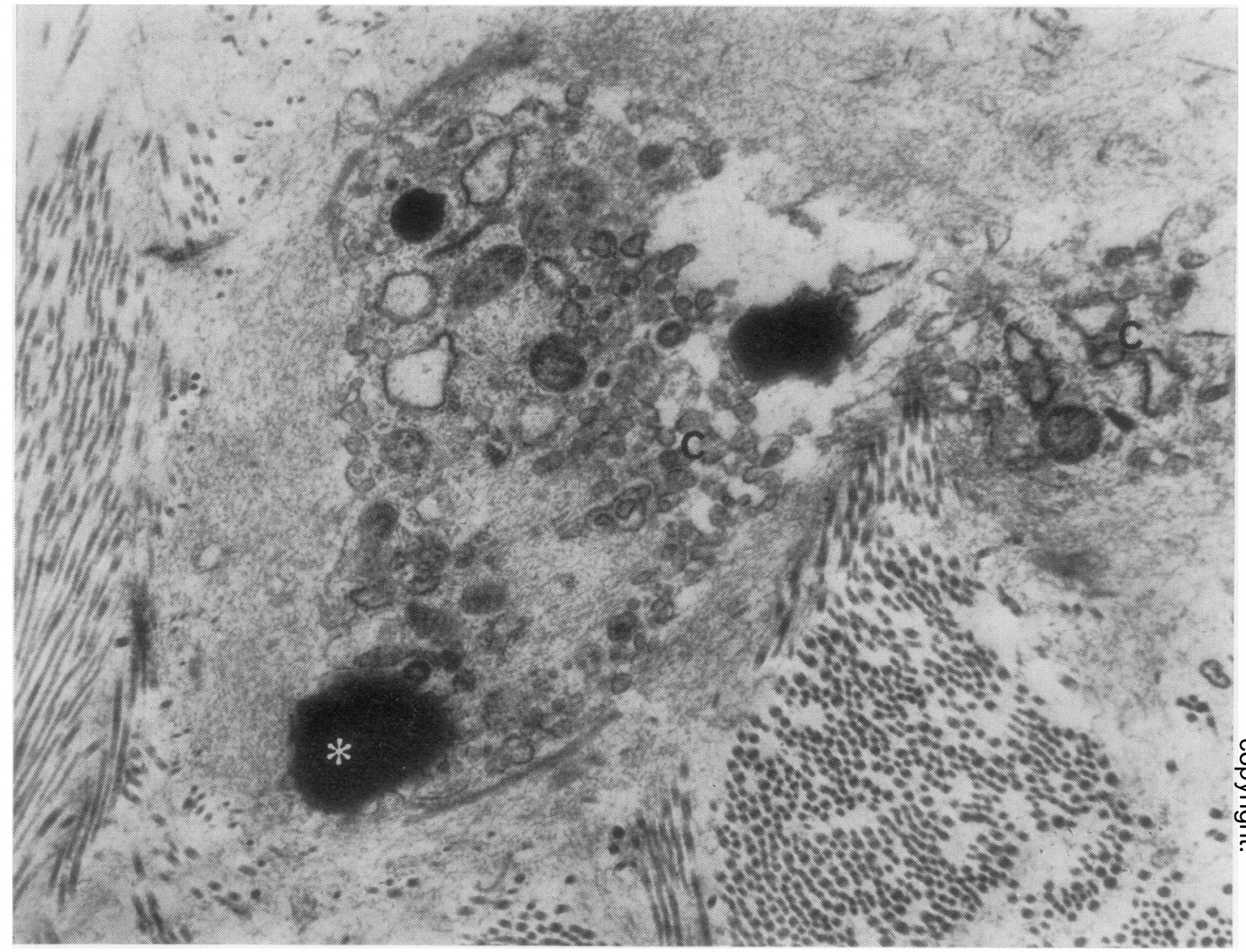

FIG. 7 In situ necrosis of chondrocyte from corn oil-injected joint. Cytomembrane remnants $(C)$ and electron dense lipid $\left(^{*}\right)$ is seen escaping into the matrix. $\times 21,000$

or that lipolysis occurs and the component parts traverse the matrix and resynthesis of the lipid occurs at some later stage, perhaps within the chondrocyte.

It should be noted, however, that injected lipid was occasionally seen lying in the matrix but, since this invariably presented as relatively large droplets accompanied by cellular debris and cytomembrane remnants derived from in situ necrosis of chondrocytes, it is clear that this is injected lipid liberated from disintegrated chondrocytes and not lipid on its passage from joint space to the cell.

The manner in which chondrocytes take up lipid is difficult to determine by electron microscopy. However, the idea that lipid may be taken into the chondrocytes by pinocytosis or micropinocytosis finds no support in the present study with corn oil or in past study with autologous fat. Micropinocytotic vesicles were in fact scanty as compared to the normal and cell processes were folded rather than extended into the matrix. On the other hand the idea that lipolysis occurs and is followed by uptake of the component parts, and resynthesis of the lipid within the cell is supported by the finding of electron dense material presumably lipidic in nature in the Golgi complex. This is in keeping with the observation that, when autologous fat is injected into the joint, markedly lucent material is demonstrable in this region (Ghadially and others, 1970).

Thus the collective evidence from our studies with autologous fat and corn oil suggests that lipolysis occurs and that the component parts traverse the cartilage matrix, and are ultimately picked up by the chondrocytes and resynthesized into lipid which then appears as droplets in the cytoplasmic matrix. Such a concept is gratifying for there is now considerable evidence that hydrolysis of triglycerides occurs before uptake by many tissues, such as adipose, mammary, and hepatic tissue and cardiac and skeletal muscle (Stein and Stein, 1967; Scow, 1970).

Necrosis of chondrocytes in situ was seen much more frequently in injected than in control joints, and at times an appearance thought to be early fibrillation of the surface was detected. Similar changes have been noted by us in joints injected with autologous fat also. In this respect corn oil seems to be somewhat more noxious than autologous fat, but the significance 


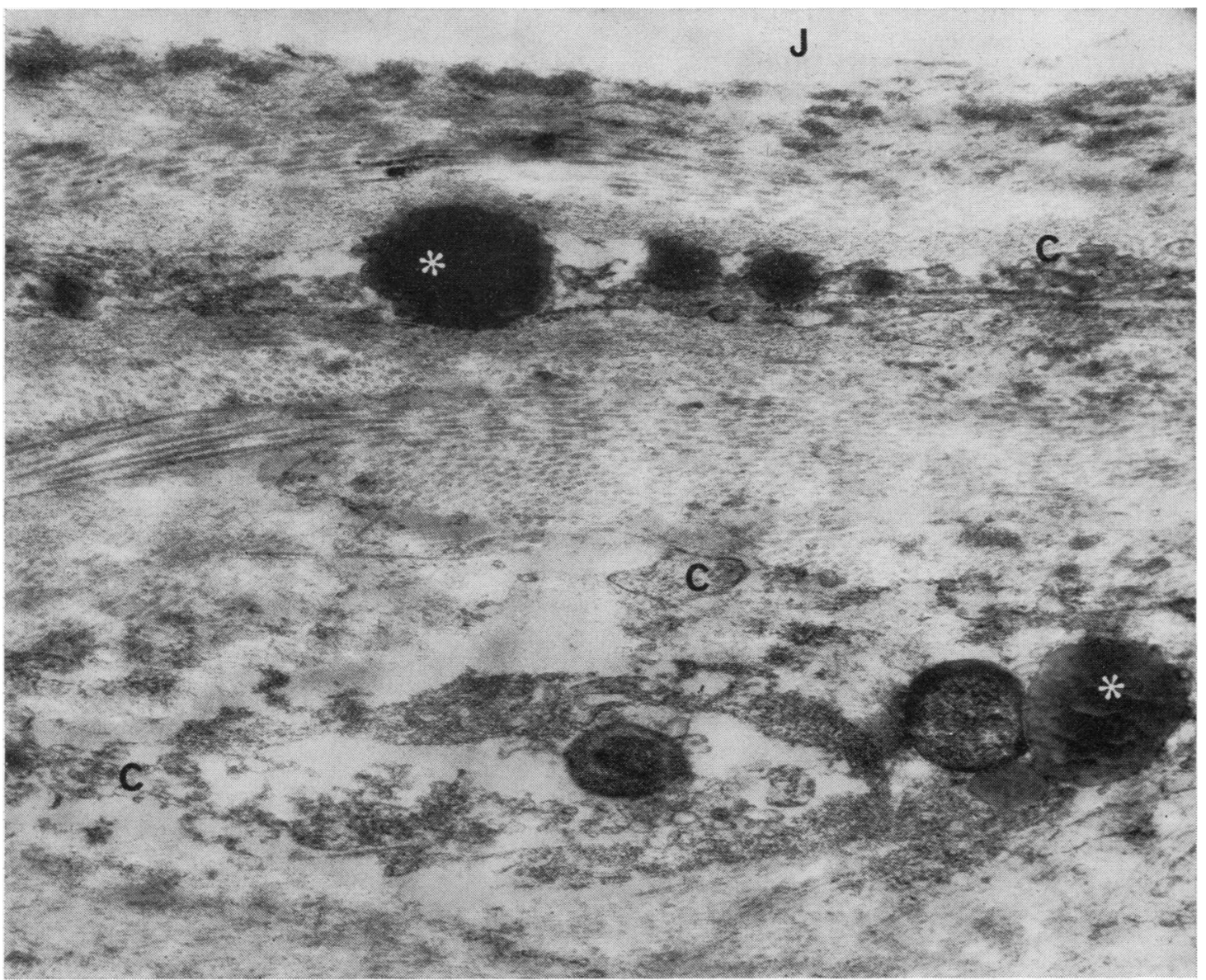

FIG. 8 Articular cartilage from corn oil-injected joint showing electron dense lipid $\left(^{*}\right)$ admixed with cytomembrane remnants $(C)$ lying free in the matrix (joints space $J$ ). $\times 26,000$

of these changes is not quite clear. It it possible that a film of oil at the articular surface may interfere with the exchange of metabolites between synovial fluid and articular cartilage and thus effect the health of chondrocytes.

\section{Summary}

Lipoarthrosis was produced in the rabbit by an intraarticular injection of corn oil. The lipid readily entered the articular cartilage and was found in chondrocytes as droplets of variable size and medium to high electron density. There is evidence which suggests that the lipid is first hydrolysed and that the components are then taken up and resynthesized by the chondrocyte. In situ necrosis of chondrocytes was seen much more frequently in injected than in control joints. The disintegration of such necrotic cells liberated the lipid into the matrix. The surface of cartilage was uneven and at times appearances suggesting early fibrillation were seen.

This work was supported by grants to one of us (F. N. G.) from the Canadian Arthritis and Rheumatism Society and the Medical Research Council of Canada.

\section{References}

Barnett, C. H., Cochrane, W., And Palfrey, A. J. (1963) Ann. rheum. Dis., 22, 389 (Age changes in articular cartilage of rabbits)

Collins, D. H., Ghadially, F. N., ANd Meachim, G. (1965) Ibid., 24, 123 (Intracellular lipids of cartilage)

Davies, D. V., Barnett, C. H., Cochrane, W. and Palfrey, A. J. (1962) Ibid., 21, 11 (Electronmicroscopy of articular cartilage in the young adult rabbit)

FAwCETt, D. W. (1966) 'The Cell, Its Organelles and Inclusions', p. 307. Saunders, Philadelphia 
Ghadially, F. N., Janzen, H. K., ANd Mehta, P. N. (1970) Arch. Path., 89, 291 (Synovial membrane in experimental lipoarthrosis)

-, Meachim, G., AND Collins, D. H. (1965) Ann. rheum. Dis., 24, 136 (Extracellular lipid in the matrix of human articular cartilage)

—, Mehta, P. N., AND KiRKaldy-Willis, W. H. (1970) J. Bone Jt Surg., 52-A, 1147 (Ultrastructure of articular cartilage in experimentally produced lipoarthrosis)

- AND RoY, S. (1967) Ann. rheum. Dis., 26, 117 (Histochemistry of synovium in experimental haemarthrosis in the rabbit)

- (1969a) 'Ultrastructure of Synovial Joints in Health and Disease', p. 105. Butterworths, London (1969b) Ann. rheum. Dis., 28, 529 (Ultrastructural changes in the synovial membrane in lipohaemarthrosis)

Hoaglund, F. T. (1967) J. Bone Jt Surg., 49-A, 285 (The response of canine knees to injections of autologous blood)

KEY, J. A. (1929) Ibid., 11, 705 (Experimental arthritis. The reactions of joints to mild irritants)

(1932) Ann. Surg., 95, 198 (Haemophilic arthritis. Bleeder's joints)

Palfrey, A. J., AND Davies, D. V. (1966) J. Anat. (Lond.), 100, 213 (The fine structure of chondrocytes)

Richardson, K. C., JARETT, L., AND FinKe, E. H. (1960) Stain Technol., 35, 313 (Embedding in epoxy resins for ultrathin sectioning in electron microscopy)

Roy, S., ANd Ghadially, F. N. (1966) Ann. rheum. Dis., 25, 402 (Pathology of experimental haemarthrosis)

- (1967) J. Bone Jt Surg., 49-A, 1636 (Ultrastructure of synovial membrane in human haemarthrosis)

_- (1969) Ann. rheum. Dis., 28, 402 (Synovial membrane in experimentally-produced chronic haemarthrosis)

Scow, R. O. (1970) 'Transport of tryglyceride. Its removal from blood circulation and uptake by tissues', in 'Parenteral Nutrition', ed. H. C. Meng and D. H. Law, p. 294. Thomas, Springfield

Stein, O., AND STeIN, Y. (1967) Lab. Invest., 17, 436 (The role of the liver in the metabolism of chylomicrons. Studied by electron microscopic autoradiography)

StoeCKEnIUS, W., AND MAHR, S. C. (1965) Ibid., 14, 458 (Studies on the reaction of osmium tetroxide with lipids and related compounds) 